\title{
Kybele in Griechenland - Ankunft und Aufnahme der Göttermutter in der griechischen Welt
}

\author{
Sabine Viktoria Kofler \\ Kerngebiet: Alte Geschichte \\ eingereicht bei: Mag. Dr. Werner Petermandl \\ eingereicht im Semester: SoSe 2016 \\ Rubrik: SE-Arbeit
}

\section{Abstract \\ Cybele in Greece. The Arrival and Reception of the Mother of the Gods in the ancient Greek world}

The following paper aims to show the arrival and reception of the Phrygian Mother Goddess Matar in the Greek world. It will first take a closer look at the early known sources, be it written or archaeological, of the Goddess Kybele. This article will further present, on the basis of these sources, the way the Mother of the Gods took from ancient Phrygia across Anatolia to Athens. Through her journey into the Greek world Kybele had gone through an essential transformation to be accepted and worshipped as one of the primary Mother Goddess' of the ancient world.

\section{Einleitung}

Matar, Meter Theon, Kybele oder Magna Mater, die große Muttergöttin der antiken Welt hatte viele Namen. Dank ihrer Popularität wurde sie jahrhundertelang verehrt. Sie wurde von denjenigen, die ihren Kult übernahmen, verändert und an jeweils eigene Bedürfnisse angepasst. Eine phrygische Matar des 8. Jahrhunderts v. Chr. hatte mit der römischen Magna Mater in augusteischer Zeit wenig gemeinsam und doch lebte die Große Mutter über Kulturen und Jahrhunderte hinweg im religiösen Bewusstsein der Menschen fort. Ein Teil des Weges, den diese Göttermutter in der antiken Welt nahm, soll in dieser Seminararbeit behandelt werden. 
Folgende Forschungsfragen werden in dieser Arbeit gestellt: Wie wurde Kybele in Griechenland aufgenommen und wie hat sie sich im Vergleich zu ihrem Vorbild, der phrygischen Matar, verändert? Welchen Transformationen unterlag diese Göttin schlussendlich auf ihrer Reise nach Griechenland? Die These dazu lautet, dass durch eine Spezifizierung von Kybeles ikonographischen Merkmalen und einen Synkretismus mit bekannteren griechischen Muttergöttinnen, Kybele erfolgreich in das griechische Pantheon integriert werden konnte.

Die Ankunft und Aufnahme von Kybele in der griechischen Welt ist ein spannendes und breit angelegtes Forschungsfeld. Bei der Fülle an Forschungsmaterial ist es sinnvoll, eine zeitliche und räumliche Eingrenzung vorzunehmen. Die zeitlichen und räumlichen Rahmen in dieser Arbeit werden deshalb die griechische Welt, die Küstenstädte in Kleinasien zu Beginn des 7. Jahrhunderts v. Chr., in denen der Kybelekult archäologisch fassbar ist und das Athen des 4. Jahrhunderts v. Chr., aus dem uns schriftliche Überlieferungen bekannt sind, umfassen.

Als Quellen für Kybeles Ankunft dienen in erster Linie schriftliche Zeugnisse von antiken Autoren, hier in deutscher und englischer Übersetzung angeführt und substantielle archäologische Funde. Dabei muss eine Auswahl von den aussagekräftigsten Quellen durchgeführt werden, zumal eine Bearbeitung des gesamten vorhandenen Materials den Rahmen dieser Arbeit sprengen würde.

Da das Forschungsgebiet so facettenreich und in Teilen auch noch nicht vollständig bearbeitet ist, gehen die Meinungen der Wissenschaftler_innen bisweilen weit auseinander. Daher werden in dieser Arbeit auch kontrovers diskutierte Thesen und Theorien berücksichtigt. Ein solches Beispiel ist etwa die weitreichende Diskussion über Kybeles und Matars Herkunft. Sie bezeugt, dass hier noch kein vollständiger Konsens zwischen den Forscher_innen und ein Forschungsdesiderat bestehen.

Eine der wichtigsten neueren Arbeiten zu Kybele ist das Werk der Archäologin Lynn E. Roller.' Ihre Arbeit kann zweifellos als das Standardwerk zur Göttermutter in der antiken Mittelmeerwelt angesehen werden. Rollers Fokus liegt dabei auf der phrygischen Matar, kommt in ihrem Werk aber auch ausführlich auf die griechischen und römischen archäologischen Quellen für Kybele zu sprechen. Maria Xagorari-Gleißner beschäftigt sich hingegen hauptsächlich mit der Göttermutter in Griechenland und stellt einige interessante Gegenthesen zu Rollers Werk auf.2 Friederike Naumanns Werk über die Ikonographie der Kybele in Phrygien und Griechenland ist zwar schon etwas älter, leistet allerdings einen wichtigen Beitrag durch ihre umfangreiche Auflistung und Beschreibung aller bis dahin bekannten Kybelestatuen und Votive. ${ }^{3}$

Lynn E. Roller, In Search of God the Mother. The Cult of Anatolian Cybele, Berkeley-Los Angeles-London 1999.

Maria Xagorari-Gleißner, Meter Theon. Die Göttermutter bei den Griechen (Peleus Studien zur Archäologie und Geschichte Griechenlands und Zyperns 40), Mainz-Ruhpolding 2008.

3 Friederike Naumann, Die Ikonographie der Kybele in der phrygischen und der griechischen Kunst (Istanbuler Mitteilungen Beiheft 28), Tübingen 1983. 
Bevor Kybele nach Griechenland kam, war sie als Matar in Phrygien bekannt. In einem ersten Kapitel wird daher ein Blick auf diese phrygische Gottheit geworfen. Im nächsten Teil der Arbeit werden die frühesten schriftlichen und archäologischen Quellen zu Kybele in Griechenland und deren Interpretationen durch die Forscher_innen aufgezeigt. Die Abbildungen von Kybele sind die maßgeblichen Quellen, die uns über die Göttin bei den Griechen, ihre Kulte und Anhänger_innen, Auskunft geben. Abschließend wird die Aufnahme der Kybele anhand ihrer Assimilation zu anderen Göttinnen untersucht, die bei ihrer Ankunft in den griechischen Städten erfolgte. In einem Resümee werden nochmals die wichtigsten Erkenntnisse und Ergebnisse zusammengefasst.

\section{Die Große Göttermutter in Phrygien}

\subsection{Die phrygische Heimat von Matar}

Ursprünglich stammte die griechische Kybele aus Phrygien, wo sie Matar, das phrygische Wort für Mutter, genannt und verehrt wurde. Ihre phrygische Herkunft war schon den griechischen Geschichtsschreibern bekannt. So schrieb zum Beispiel Diodor in seiner Weltgeschichte über die Große Mutter: „Allerdings wird auch überliefert, diese Göttin sei in Phrygien geboren." Diese antike Landschaft lag in der heutigen Türkei, im westlichen Kleinasien. Laut Herodot wanderten die frühen Phrygier_innen aus Europa, dem Balkangebiet, ein und brachten von dort ihre Kulte mit. „Die Phryger heißen, wie die Makedonen sagen, Briger, solange sie in Europa mit den Makedonen zusammenwohnten. Bei ihrem Übergang nach Asien änderten sie mit ihrem Land auch ihren Namen in Phryger. ${ }^{\prime 5}$ Die Frage der Herkunft der Phryger_innen hat schon moderne Forscher_innen aufgrund dieser Aussage von Herodot beschäftigt. Maya Vassileva untersucht derzeit die überaus interessante Verbindung von bulgarischen und phrygischen Steinmonumenten und Gräbern, die auf eine frühere Verwandtschaft der beiden Völker hinweisen könnte. ${ }^{6}$ Archäologisch fassbar sind die Phrygier_innen in ihrem Siedlungsgebiet frühestens im 9. Jahrhundert v. Chr. Im 8. Jahrhundert v. Chr. bildete sich ein phrygisches Königreich mit dem Kernland um die Hauptstadt Gordion, am Fluss Sangarios, heraus.?

Seine kulturelle und politische Blütezeit hatte das phrygische Reich unter dem noch in griechisch-römischer Literatur sowie in assyrischen Quellen bekannten König Midas. ${ }^{8}$ Der von Legenden und Mythen umwobene König soll neben der Einführung der

4 Diodorus Siculus, Griechische Weltgeschichte (Bibliothek der griechischen Literatur 34), Buch I-X. Teil 1: Buch I-III, übersetzt von Gerhard Wirth/Otto Veh, Stuttgart 1992, S. 3, Sp. 58, Absatz 1.

5 Herodot, Historien, Griechisch-Deutsch, herausgegeben von Josef von Feix (Sammlung Tusculum), Düsseldorf 2006, S. 7, Sp. 73.

6 Maya Vassileva, Further Considerations on the Cult of Kybele, in: Anatolien Studies 51 (2001), S. 51-63, hier S. 55, [http://www.jstor.org/stable/3643027], eingesehen 11.7.2016.

7 Eckart Olshausen, Phryges, Phrygia, in: Der Neue Pauly, Bd. 6, Stuttgart-Weimar 1999, Sp. 965-967, hier Sp. 966

8 Anne-Maria Wittke, Kleinasien, in: Der Neue Pauly 2006, [http://dx.doi.org/10.1163/1574-9347_dnp_e614980], eingesehen 4.8.2016. 
phrygischen Schrift auch den Kult für die Große Mutter begründet haben. ${ }^{9}$ In der sogenannten Midasstadt und deren Umgebung im phrygischen Hochland wurden die meisten Denkmäler für die Große Mutter gefunden, weshalb es sich wohl um ein Kultzentrum für die Göttin handelte. ${ }^{10}$

Als die ältesten bildlichen Darstellungen von der phrygischen Matar werden generell Felsmonumente im phrygischen Hochland betrachtet, die aus dem 8. Jahrhundert v. Chr. stammen. Diese Monumente aus Stein ahmen die Form von Gebäudefronten mit einem Giebel nach. Ihre Fassaden sind mit geometrischen Mustern überzogen und weisen in der Mitte eine Nische - in Form eines Türeinganges - für das Kultbild der Göttin auf. Aus altphrygischen Inschriften, wie dem Midasmonument aus dem späten 8. Jahrhundert v. Chr., können diese Steinmonumente eindeutig der phrygischen Matar zugeordnet werden, auch wenn die Kultstatue in manchen Felsdenkmälern fehlt. ${ }^{11}$ Ein anschauliches Beispiel für ein solches Felsmonument ist jenes in Arslankaya. Es ist das größte und besterhaltene Kultbild der Matar, das auf das Ende des 7. Jahrhunderts v. Chr. datiert wird. ${ }^{12}$ Auf der sieben Meter hohen Gebäudefassade des Monumentes befindet sich in der Mitte ein Eingang zur Nische mit dem Kultbild der Göttin. Eine nennenswerte Besonderheit bilden die zwei Tierreliefs an der Seite der Göttin in der Nische. Es sind zwei aufrecht stehende Löwen, die sich der Göttin zuwenden. ${ }^{13}$ Löwen als Begleittiere der Matar sind aus vorgriechischer Zeit in Phrygien nur auf diesem Monument bezeugt, sie sind damit also keine exklusiven Merkmale für Matar. ${ }^{14}$ Die Vertiefung im Felsen, in der das Kultbild der Göttin steht, wird als eine Art Eingang zum Haus der Göttin interpretiert, das wiederum mit der Vorstellung von den Bergen als Domizil und Herrschaftsbereich der Matar verbunden werden kann. ${ }^{15}$ Solche Steinmonumente finden sich in ganz Phrygien, meist an einzeln stehenden herausragenden Felsen oder in den großen Kultzentren, wie der Midasstadt. ${ }^{16}$ Weitere Abbildungen von Matar tauchen als Statuetten für den privaten Kult der phrygischen Bevölkerung und auf öffentlichen Stelen mit Matarreliefs auf.17

Bei der Erforschung der Kultpraktiken zu Matar aus vorgriechischer Zeit muss wegen des Mangels an schriftlichen auf die archäologischen Quellen verwiesen werden. Die Steinmonumente könnten möglicherweise als Kultplätze für Rituale gedient haben, von denen wir jedoch bislang wenig wissen. Aus Darstellungen auf Reliefs lassen sich die Verwendung von Musikinstrumenten und eventuell auch Tänze ableiten. Schriftliche Quellen für phrygische Kulte, wie etwa die ekstatischen Tänze und Selbst-

9 Peter Högemann, Midas, in: Der Neue Pauly 2006, [http://dx.doi.org/10.1163/1574-9347_dnp_e803950], eingesehen 4.8.2016; Vassileva, Further Considerations, S. 53.

10 Naumann, Die Ikonographie der Kybele, S. 41.

11 Mary Jane Rein, Phrygian Matar. Emergence of an Iconographic Type, in: Eugene N. Lane (Hrsg,), Cybele, Attis and Related Cults. Essays in Memory of M. J. Vermaseren, (Religions in the Graeco-Roman World 131), Leiden-New York-Köln 1996, S. 223-237, hier S. 224.

12 Naumann, Die Ikonographie der Kybele, S. 43 und S. 61.

13 Ebd., S. 43-44

14 Brigitte Bøgh, The Phrygian Background of Kybele, in: Numen 54 (2007), Heft 3, S. 304-339, hier S. 324, [http:// www.jstor.org/stable/27643268], eingesehen 12.7.2016.

15 Naumann, Die Ikonographie der Kybele, S. 55; Rein, Phrygian Matar, S. 234.

16 Naumann, Die Ikonographie der Kybele, S. 54.

17 Bøgh, The Phrygian Background, S. 326-328. 
kastrierung von Priestern sind uns erst aus griechisch-römischer Zeit erhalten und spiegelten wohl eher die zeitgenössischen Vorstellungen der Autoren von Kybelekulten und den Mysterien der Göttin wider. ${ }^{18}$

Matar bleibt damit die einzige Gottheit aus altphrygischer Zeit, von der wir anthropomorphe Darstellungen haben. ${ }^{19}$ Ein Grund dafür könnte auf Einflüsse von benachbarten Kulturen, wie den Hethiter und Urartäer, die bereits menschenähnliche Göttergestalten kannten, zurückzuführen sein. ${ }^{20}$ Beeinflussung durch Kulturkontakte gab es auch in der ikonographischen Darstellung von Matar durch die hethitische Göttin Kubaba. Diese Ähnlichkeiten ließen Forscher_innen vermuten, die phrygische Matar sei eine Nachfolgerin dieser Göttin. Die Herkunft von Matar wurde in der Tat schon länger diskutiert und im Folgenden sollen die bekanntesten Theorien dazu kurz vorgestellt werden.

\subsection{Herkunftstheorien zu Matar}

Die Bezeichnung Matar ist in phrygischen Inschriften zehn Mal bezeugt, zwei Mal mit dem Beiwort kubileya, was übersetzt „von dem Berg“ bedeutet. Daraus entwickelte sich der griechisch-römische Name Kybele/Cybele für die Göttin. ${ }^{21}$

Durch die Entzifferung der hethitischen Hieroglyphen in den 1920er-Jahren glaubte als erster moderner Forscher William Albright eine linguistische Ähnlichkeit zwischen den Namen Kubaba und Kybele bzw. den Beinamen von Matar, kubileya gefunden zu haben. Auch in Lexika wird Kubaba oft als eine Art Vorform von Kybele angegeben. ${ }^{22}$ Die langen kulturellen und politischen Kontakte zwischen Hethiter_innen und Phrygier_innen könnten als Hinweis auf eine phrygische Aufnahme von Kubaba in die eigene Götterwelt gedeutet werden. Kubaba war seit dem 18. Jahrhundert v. Chr. Stadtgöttin von Karkemish, einer an der heutigen syrischen Grenze zur Türkei gelegenen Stadt. ${ }^{23}$ Ikonographisch besteht ein sichtlicher Einfluss auf die Abbildungen der phrygischen Matar. Kubaba und Matar wurden auf Stelen frontal innerhalb eines Rahmens, ähnlich den Felsmonumenten, dargestellt. Kubabas Begleittier, der Falke, ist auch ein bevorzugtes Motiv in der Matar-Ikonographie. ${ }^{24}$ Allerdings fehlt Kubaba die wichtigste Eigenschaft, die sie mit der phrygischen Matar verbinden würde. Sie ist zwar die Herrin des Lebens, der Fruchtbarkeit und der wilden Tiere, allerdings keine Muttergöttin. ${ }^{25}$ Obwohl es einige Ähnlichkeiten in der Ikonographie von Kubaba und Matar gibt, fehlt innen ein eindeutiges Identifikationsmerkmal, dass es sich um dieselbe Göttin handelt. Es besteht durchaus die Möglichkeit, dass die Ikonographie der Kubaba auf die Matar-

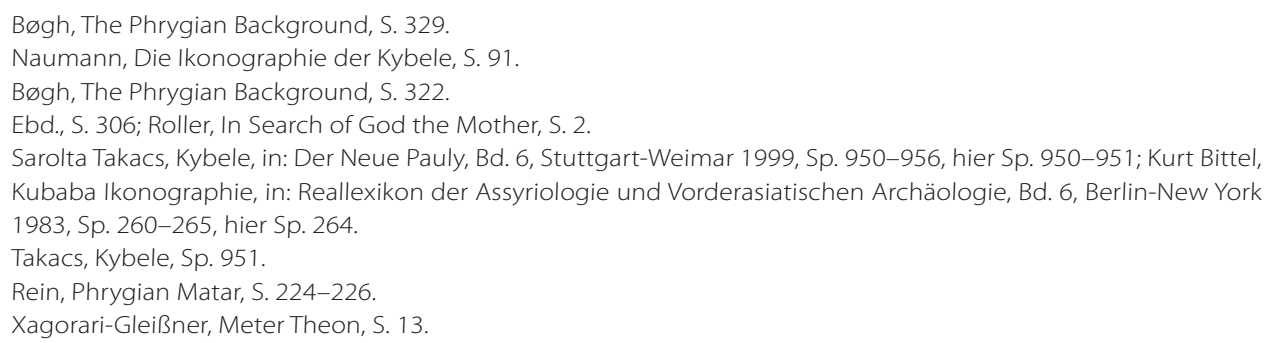


reliefs der Phrygier Einfluss hatte, ohne dass sie zwingend ihre Vorgängerin war. In der Forschung wird die Theorie einer Kubaba-Kybele Abstammung oder Gleichsetzung der beiden jedoch noch immer diskutiert.

Eine sehr populäre Theorie, auch in Nicht-Fachkreisen, ist die Vorstellung einer prähistorischen, universalen Muttergottheit, die in historischen Zeiten in uns bekannte Göttinnen verwandelt wurde. ${ }^{26}$ Anhänger_innen dieser Theorie sind der Meinung, die Anbetung einer Muttergottheit in der Frühzeit der Menschheitsgeschichte sei die erste Form von Religion überhaupt gewesen. ${ }^{27}$ Als Beweisquelle werden kleine Statuetten mit weiblichen Figuren aus neolithischer Zeit herangezogen. Diese Figuren sollten eine Fruchtbarkeitsgöttin mit ausgeprägtem mütterlichem Aspekt darstellen. Matar sei dieser Theorie zufolge eine Nachfahrin dieser ursprünglichen Muttergöttin. Allerdings weist diese Interpretation eine Reihe von Lücken auf, denen Lynn Roller genauer nachgeht. Sie macht besonders auf den Fundkontext dieser Figuren aufmerksam, der nicht ausschließlich für einen religiösen Zweck solcher Gegenstände spricht. Die meisten dieser Objekte wurden in Abfalllagern von Haushaltsgegenständen gefunden und lassen auch andere Interpretationen für deren Verwendungszweck zu, beispielsweise als Lernmaterial, Spielzeug oder auch für magische Praktiken. ${ }^{28}$

Nichtsdestotrotz hat die Vorstellung einer vorzeitlichen Muttergottheit und deren Relevanz in einer Gesellschaft schon manche frühe Forscher beschäftigt, wie etwa den bekanntesten darunter, Johann Jakob Bachofen. Als der Baseler Gelehrte 1861 sein Werk „Das Mutterrecht"29 veröffentlichte, stießen seine Thesen bei seinen Zeitgenoss_ innen vorwiegend auf Skepsis und Ablehnung. ${ }^{30}$ Seinen Ausführungen zufolge machte die Geschichte der Menschheit mehrere Entwicklungsstufen durch, von denen eine Periode das sogenannte "gynaikokratische Weltalter"31 gewesen sei. Es herrschte also in einigen menschlichen Gesellschaften ein „Mutterrecht", in heutiger Terminologie ein Matriarchat, das in griechischer und römischer Zeit von einem „Vater- oder Männerrecht", dem Patriarchat, in einem mythologischen und für Bachofen realen Kampf der Geschlechter abgelöst wurde. ${ }^{32}$ Bachofens Quellen waren mythische Erzählungen und Berichte antiker Autoren, wobei er keinerlei Trennung zwischen Mythen und historischer Realität vollzog. Das Mutterrecht war für Bachofen eine historische Tatsache, obwohl er keine weiteren Beweise als seine eigenen Ausführungen liefern konnte. Er war kein Vertreter der Methode einer rationalen, objektiven Quellenkritik, wie es etwa sein Zeitgenosse Leopold von Ranke war. Seine spekulativen Interpretationen von Mythen und Sagen hätten weitaus besser in die Zeit der Romantik gepasst, weshalb er schon zu seiner Zeit auf Ablehnung und Unverständnis stieß.33

26 Takacs, Kybele, Sp. 952.

27 Bøgh, The Phrygian Background, S. 312.

28 Roller, In Search of God the Mother, S. 15; Bøgh, The Phrygian Background, S. 313.

29 Johann Jakob Bachofen, Das Mutterrecht. Eine Untersuchung über die Gynaikokratie der alten Welt nach ihrer religiösen und rechtlichen Natur, Stuttgart 1861.

30 Erik Wolf, Bachofen, Johann Jakob, in: Deutsche Biographie, o. D., [https://www.deutsche-biographie.de/ gnd1 18505645.html\#ndbcontent], eingesehen 27.3.2018.

31 Hans G. Kippenberg (Hrsg.), Johann Jakob Bachofen. Mutterrecht und Urreligion, Stuttgart 1984, S. 76.

32 Kippenberg, Johann Jakob Bachofen, S. 181.

33 Wolf, Bachofen, eingesehen 27.3.2018. 
Für Bachofen war das Volk der Lykier_innen, nach Herodots Beschreibung, eine vom Mutterrecht geprägte Gesellschaft, die es auch in anderen "Stämmen" und "Völkern” der antiken Welt gab. Wirft man jedoch einen genaueren Blick auf Herodots Ausführung über die Lykier_innen, kann von einem antiken Matriarchat nicht die Rede sein:

„Einen Brauch aber pflegen sie für sich, der sich sonst nirgends auf der Welt findet: Sie nennen sich nach ihren Müttern, nicht nach den Vätern. Fragt man einen Lykier nach seiner Herkunft, dann nennt er den Namen seiner Mutter und zählt ihre weiblichen Vorfahren auf."34

Was Herodot hier beschreibt ist die Matrilinearität, also die Abstammung nach der Mutter, allerdings ist dies für das historische Volk der Lykier_innen nicht nachweisbar. ${ }^{35}$ Bachofen schließt fälschlicherweise von einer scheinbaren matrilinearen Gesellschaft auf ein Matriarchat, eine Herrschaft der Frauen, die es so nie gegeben hat. ${ }^{36}$ Diesem Matriarchat steht ein weibliches Prinzip, "die große Mutter", gleichbedeutend mit der Mutter Erde als Ursprung allen Lebens zugrunde. ${ }^{37}$ Für Bachofen war dieser Glaube an eine ursprüngliche Muttergottheit und ein damit einhergehendes Matriarchat eine konkrete Stufe in der Menschheitsentwicklung.

Da die Lykier_innen auf anatolischem Boden lebten, genau wie später die Phrygier_innen, wurde diese Matriarchatstheorie und die Vorstellung einer frühzeitlichen Muttergottheit auf die Phrygier_innnen und Matar projiziert. Zu Beginn des 20. Jahrhunderts waren Forscher_innen für Bachofens Theorie weitaus zugänglicher, besonders in Hinblick auf erste archäologische Ausgrabungen im Siedlungsgebiet der Phrygier_innen, die eine Muttergottheit als Hauptgöttin erkennen ließen. Die These, dass die griechisch-römische Kybele und die phrygische Matar eine Nachfahrin einer urzeitlichen Muttergottheit sei, hält sich in der Forschung nach wie vor hartnäckig. ${ }^{38}$

\section{Kybeles Ankunft in der griechischen Welt}

\subsection{Von Phrygien nach Lydien}

Der Weg, den die phrygische Matar nach Griechenland nahm, ist aufgrund archäologischer Funde rekonstruierbar. Die Forschung geht allgemein davon aus, dass sich Matar im 6. Jahrhundert v. Chr. über Lydien, das südwestlich von Phrygien liegt, in den kleinasiatischen griechischen Küstenstädten und von da an weiter nach Westen ausgebreitet hatte. ${ }^{39}$ In Lydien war die Muttergöttin den Griechen jedoch unter einem anderen

34 Hdt. 1, 173.

35 Im Gegenteil, auf lykischen Gräberinschriften wurden Patronyme verwendet, was auf eine patrilineare Tradition in der lykischen Gesellschaft schließen lässt, siehe Roller, In Search of God the Mother, S. 11-12.

36 Uwe Wesel, Der Mythos vom Matriarchat. Über Bachofens Mutterrecht und die Stellung von Frauen in frühen Gesellschaften, Frankfurt am Main 1981, S. 36.

37 Bachofen, Das Mutterrecht, S. 186-187.

38 Roller, In Search of God the Mother, S. 12-13.

39 Philippe Borgeaud, Die Mutter der Götter. Von Anatolien über Griechenland nach Rom, in: Badisches Landesmuseum Karlsruhe (Hrsg.), Imperium der Götter. Isis-Mithras-Christus, Kulte und Religionen im Römischen Reich, Darmstadt 2013, S. 85-91, hier S. 85. 
Namen bekannt. Herodot schildert die Zerstörung der lydischen Hauptstadt um 499 v. Chr. und schreibt: „Sardes wurde niedergebrannt und mit der Stadt auch das Heiligtum der Stammgöttin Kybebe." ${ }^{\prime 0}$ Die Namen Kybebe und Kybele scheinen sich auf den ersten Blick sehr ähnlich zu sein und antike griechische Autoren verwendeten die Namen durchaus synonym. Sprachwissenschaftliche Untersuchungen ergeben aber ein differenziertes Bild. Im Griechischen waren sich die beiden Namen Kußńßn (Kybebe) und

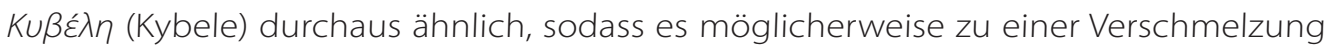
der beiden kam. In ihren anatolischen Heimatsprachen waren die Namen indes klar differenzierbar. ${ }^{41}$ Mark Munn argumentiert hingegen, dass sich Kybebe aus der vorhin schon genannten hethitischen Göttin Kubaba, in lydischer Sprache Kuvava ausgesprochen, ableitet. ${ }^{42}$ Kubaba/Kuvava wurde somit von den Griechen als Kybebe übersetzt und mit Kybele gleichgesetzt. ${ }^{43}$ Das würde erklären, warum in den frühen griechischen Quellen manchmal von Kybebe die Rede ist, wenn die Göttermutter gemeint ist. Der Name Kybebe verschwindet allerdings im späten 5. Jahrhundert v. Chr. aus der griechischen Literatur. ${ }^{44}$ Die Griech_innen nahmen Kybele also zunächst durch einen lydisch geprägten Filter auf. ${ }^{45}$ Ein Hinweis darauf zeigt sich insbesondere in der griechischen Ikonographie von Kybele, deren charakteristisches Attribut der Löwe werden sollte und eigentlich eine lydische Beifügung war, wie weiter unten noch geklärt werden wird.

Zeugnisse für eine greifbare Präsenz von Kybele bei den Griech_innen sind einige literarische Schriftquellen, die uns jedoch zum Teil nur fragmentarisch überliefert sind.

\subsection{Frühe literarische Quellen}

Kybele wird, abgeleitet von kubileya, zum griechischen Eigennamen der Göttin. Bekannter ist sie hingegen als die Meter Theon, die Göttermutter oder als Meter oreia, die "Mutter von den Bergen".46 In vorklassischer Zeit finden sich in der literarischen Überlieferung verstreut Anspielungen auf Kybele oder die Göttermutter. Die Interpretationen der schriftlichen Quellen sind allerdings problematisch, da Kybele nicht immer eindeutig identifizierbar ist. Die Griech_innen kannten zu unterschiedlichen Zeiten mehrere Muttergottheiten. Daher werde ich zunächst einen Blick auf die frühesten literarischen Überlieferungen werfen, um festzustellen, inwieweit Kybele den Griech_innen schon bekannt war und sie in das Götterpantheon aufgenommen wurde.

Als eine der ältesten literarischen Überlieferungen wird häufig der 14. Homerische Hymnus zitiert. Dieses sechszeilige Loblied an die Göttermutter entstand ca. Ende des 6. Jahrhunderts v. Chr. und lautet folgendermaßen:

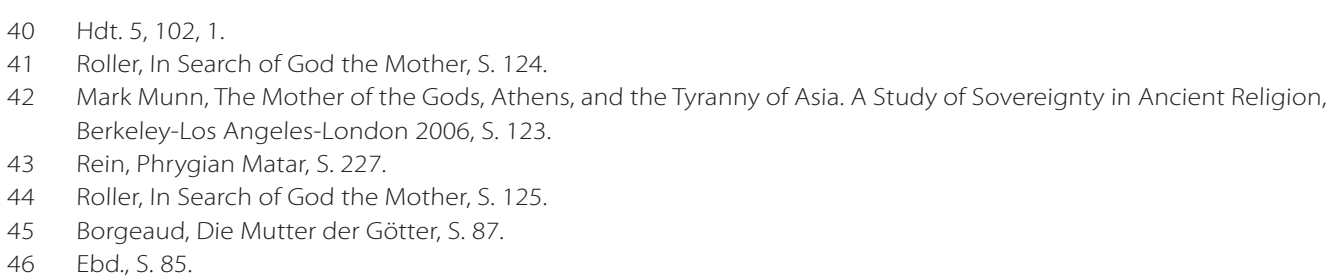


„Preise die Mutter aller zusammen, der Götter und Menschen, Muse des hellen Gesangs, des Zeus, des gewaltigen Tochter! Tosen der Klappern und Pauken, das Rauschen der Klarinetten lockt sie zur Lust, das Gebrülle von Wölfen und äugenden Löwen; Echo lockt im Gebirge, es locken Gehöfte im Walde.

Freu dich also auch du meines Sangs und ihr Göttinnen alle!"47

Die Mutter aller hat hier allerdings noch keinen Namen, es könnte sich also auch um eine andere Göttin handeln. Im 30. Homerischen Hymnus „An die Allmutter Erde“ wird Gaia in der Zeile 17 angerufen: „Heil dir, Weib des gestirnten Uranos! Göttermutter!“48 Den Stand einer Muttergöttin hatte in der griechischen Götterwelt also nicht nur ausschließlich Kybele inne. Die weiteren Beschreibungen im Lied können allerdings einen näheren Hinweis auf die Identität der Göttin bieten. Es werden Musikinstrumente, wilde Tiere und die Gebirge mit der Göttin in Zusammenhang gebracht, die auf Kybele und ihre phrygischen Wurzeln Bezug nehmen könnten. ${ }^{49}$

Die älteste literarische Überlieferung über Kybele ist bei dem Dichter Hipponax aus Ephesos um 540 v. Chr. zu finden..$^{50}$ In dem „Fragment 121 B“ wird Kybele als eine Tochter von Zeus genannt. ${ }^{51}$ Der Dichter Pindar kannte sie im frühen 5. Jahrhundert v. Chr. als "Mistress Kybele the Mother", was die bisher älteste bekannte Gleichstellung von Kybele mit dem Titel der Meter ist. ${ }^{52}$

Pindar überliefert uns ebenfalls in einem Fragment, dass er für Kybele und Pan ein Heiligtum auf seinem Besitz bauen ließ, das früheste bekannte private Heiligtum für die Göttin..$^{53}$ In einem anderen Fragment von Pindar wird Meter mit dem Gott Pan zusammen verehrt:

„Pan, Arkadiens Betreuer, der

Allerheiligsten Räume Hort!

Der Großen Mutter Begleiter,

von ehrwürdigen Huldinnen voll

Freude umhegt..."54

Der Hirtengott Pan wurde aufgrund seiner Verbindung zur wilden Natur zum Begleiter der Großen Mutter. Ähnlichkeiten in den Kultriten der beiden Gottheiten, ausgeführt durch von Ekstase oder Wahnsinn besessene Kultanhänger_innen, bestätigen eine gewisse Affinität der beiden zueinander. ${ }^{55}$ Verbindungen von Kybele zu einheimischen griechischen Göttern sind auch bei anderen Autoren belegbar. Der Historio-

47 Homer, Homerische Hymnen, Griechisch-Deutsch, herausgegeben von Anton Weiher (Sammlung Tusculum), München-Zürich 1986, S. 14.

48 Homer, Hom. h. 30.

49 Roller, In Search of God the Mother, S. 123.

50 Takacs, Kybele, Sp. 952.

51 Xagorari-Gleißner, Meter Theon, S. 15.

52 Roller, In Search of God the Mother, S. 125.

53 Xagorari-Gleißner, Meter Theon, S. 125.

54 Pindar, Siegesgesänge und Fragmente, Griechisch-Deutsch, herausgegeben von Oskar Werner, München 1967, S. 76.

55 Roller, In Search of God the Mother, S. 177. 
graph Charon von Lampsakos aus dem 5. Jahrhundert v. Chr. setzte Kybele sogar mit Aphrodite gleich. ${ }^{56}$ Eine Identifizierung von Kybele mit griechischen Göttinnen begann also schon sehr bald nach ihrer Ankunft.

Als ein weiteres Beispiel hierfür ist der berühmte "Derveni-Papyrus" Zeugnis. Der erste Papyrusfund auf griechischem Festland wird auf die Jahre 340-320 v. Chr. datiert, soll allerdings auf einen älteren schon im 6. Jahrhundert v. Chr. verfassten Text zurückgehen. Auf dem Papyrus wird unter anderem auch die orphische Kosmologie beschrieben. Darin wird in Spalte 22, Zeile 6 berichtet: „Earth (Ge), Mother (Meter), Rhea and Hera is the same (or: are one and the same)."57 In klassischer Zeit spricht Euripides in seinem Drama "Die Bakchen“ von den "mysteries of the great mother Kybele" ${ }^{48}$ und weist damit schon auf einen Mysterienkult der Göttin in Griechenland hin.

Die schriftlichen Zeugnisse aus archaischer Zeit über Kybele sind somit spärlich und geben auch kein umfassendes Bild zur griechischen Kybele und ihrem Kult wieder. Es kann allerdings festgehalten werden, dass die Griech_innen schon im 5. Jahrhundert v. Chr. von einer Muttergöttin wussten, die, erkennbar an ihrem Namen, aus Phrygien stammte. Zur Ankunft und Kultausübung der Kybele geben die schriftlichen Quellen allein kein vollständiges Bild ab, weshalb es naheliegt in einem nächsten Schritt auch die archäologischen Fundstücke bei den Griech_innen zu untersuchen, um eine eindeutigere Vorstellung über die Göttin zu erhalten.

\subsection{Die archäologischen Quellen}

Die frühesten archäologischen Zeugnisse finden sich im 6. Jahrhundert v. Chr. in den griechischen Küstenstädten Kleinasiens in Smyrna, Milet, Phokaia und Kyme. ${ }^{59}$ Durch die geographische Nähe zu Anatolien kamen diese Städte früh mit der Muttergöttin in Kontakt, wie sich aus den Funden von Statuetten und Votivgaben ableiten lässt. ${ }^{60}$ Die ältesten bildlichen Darstellungen der Kybele in den griechischen Städten sind sogenannte Naiskoi, kleine tempelförmige Kultbilder, die als Votivgaben benutzt wurden. Das ikonographische Vorbild für diese Kybelenaiskoi dürften phrygische Stelen und die Steinmonumente gewesen sein..$^{61}$ Die Nachahmung eines kleinen Tempels, in dessen Mitte die Göttin stand, ist besonders in Milet häufig zu finden. ${ }^{62}$

Eine neue Form war das Bild der thronenden Göttin im Naiskos, die vor allem ihre bestimmende Form in der klassischen Ikonographie werden sollte. Für eine Muttergöttin, die frontal auf einem Thron sitzend dargestellt wurde, gibt es in vorgriechischer Zeit keine bekannten phrygischen Vorbilder. Sie ist somit eine griechische Entwicklung. ${ }^{63}$

56 Xagorari-Gleißner, Meter Theon, S. 15.

57 Gabor Betegh, The Derveni Papyrus. Cosmology, Theology and Interpretation, Cambridge 2005.

58 Euripides, Bacchae, The Plays of Euripides, Bd. 9, Griechisch-Englisch, übersetzt und eingeleitet von Richard Seaford, herausgegeben von Christopher Collard Warminster 1996, S. 78-79.

59 Fritz Graf, The Arrival of Cybele in the Greek east, in: János Harmatta (Hrsg,), Acts du Vlle Congrès de la Fédération Internationale des Associations d’Études Classiques I, Budapest 1984, S. 117-120, hier S. 117.

60 Rein, Matar Theon, S. 229.

61 Ebd., S. 223.

62 Naumann, Die Ikonographie der Kybele, S. 111.

63 Takacs, Kybele, Sp. 952. 
Auch der Löwe sitzt immer öfter an Kybeles Seite oder liegt auf ihrem Schoß. Die meisten dieser Votive stammen aus dem ionischen Raum, den Inseln Samos und Chios sowie aus den Kolonien der griechischen Städte. ${ }^{64}$ Ihre Darstellungen zeigen, erkennbar an der Kleidung, Chiton und Mantel mit Schleier, sowie der Pose, schon eine hellenisierte Kybele. ${ }^{65}$

Eine besondere Form bildeten die Doppelnaiskoi von Kybele. In zwei Nischen wurden nebeneinander zwei weibliche Figuren abgebildet. Diese Doppelnaiskoi geben der Forschung allerdings noch einige Rätsel auf. Kybele kann anhand ihrer Attribute, Löwe, Opferschale und Tympanon, eindeutig identifiziert werden. Allerdings ist nicht sicher, wen die zweite Figur darstellen soll. Es gibt dazu verschiedene Interpretationen: Ihr Bild zweifach darzustellen wäre ein Ausdruck der Verdoppelung der Macht der Göttin. ${ }^{66}$ Wäre hingegen die zweite Statue eine andere griechische Göttin, wie Demeter oder Persephone, könnte es ein Hinweis auf eine Verbindung der phrygischen Muttergöttin mit einer einheimischen griechischen Göttin sein. Diese Interpretation folgt der Argumentationslinie, dass eine fremde Gottheit nicht-griechischen Ursprunges, die vergleichsweise rasch eine wichtige Stellung in Kult und Leben der Bevölkerung übernahm, nicht ohne eine starke Verbindung zu einer hiesigen Göttin akzeptiert werden konnte. ${ }^{67}$ Eine weitere beachtenswerte These liefert der griechische Archäologe lonnis N. Svoronos, welcher der Meinung ist, beide Figuren würden zwei verschiedene Aspekte der gleichen Göttin repräsentieren. Eine Abbildung würde auf die phrygische, fremde Muttergöttin und deren Herkunft verweisen, die andere hingegen, die nunmehr bei den Griechen heimisch gewordene athenische Muttergöttin darstellen. Diese Interpretation würde eine komplette Assimilation an eine andere Gottheit ausschließen. Argumentiert wird hier, dass auch in Rom die Magna Mater eine solche Dualität innehatte. Sie galt nach ihrer Einführung als die Mutter aus Phrygien bzw. Pessinus und zugleich auch als eine Art trojanische Vorfahrin der Römer. ${ }^{68}$

Diese Naiskoi hatten unterschiedliche Funktionen, waren aber meistens als Votive für Heiligtümer, wie Inschriften darauf belegen, gedacht. ${ }^{69}$ Die Anzahl der Kybelenaiskoi nahm im 5. und 4. Jahrhundert v. Chr. stetig zu, was von einer hohen Popularität des Kultes in der griechischen Welt zeugt. ${ }^{70}$ Votive und Statuetten von der sitzenden Kybele im Naiskos mit einem Löwen auf dem Schoß breiteten sich vom Osten der griechischen Welt bis weit in den Westen, zuerst aufs Festland, nach Athen, dem Peloponnes und Sparta sowie den griechischen Kolonien in Unteritalien und Südfrankreich aus. ${ }^{71}$

Kybeles visuelle Darstellung in der griechischen Welt hat sich im Laufe des 5. Jahrhunderts v. Chr. gefestigt und standardisiert. Athen hatte bei der Verbreitung des Kultes der Muttergöttin und ihres Bildes in der restlichen griechischen Welt eine Vorreiterrolle. Der

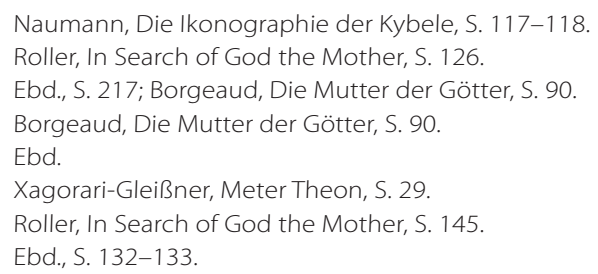


Grund war wohl die offizielle Aufnahme der Göttin in den griechischen Götterkult der Polis. Da es dafür kein fixes Datum und Ereignis, wie bei der Einführung von Kybele in Rom gibt, erzählt eine Legende von der Ankunft, anfänglichen Ablehnung und letztendlichen Akzeptanz der Göttermutter bei den Athener_innen. Überliefert ist diese Erzählung erst aus viel späterer Zeit, unter anderem auch bei Kaiser Julian aus dem 4. Jahrhundert und Photios aus dem 9. Jahrhundert. ${ }^{72}$ Darin kam ein phrygischer Priester der Kybele, gallus mètragyrtēs oder einfach der phrygische Mann genannt, nach Athen und versuchte unter den athenischen Frauen Anhängerinnen für seinen fremden Kult zu gewinnen. Erzürnt über dessen Vorhaben töteten ihn die Athener, woraufhin die Stadt von einer Plage heimgesucht wurde. Ein Orakel bestimmte, dass für den Tod des Priesters eine Buße dargebracht werden musste, um den Zorn der Göttin zu mildern. Daraufhin wurde von den Athener_innen ein Metroon, ein Tempel für die Mutter, ${ }^{73}$ errichtet. Von diesem Zeitpunkt an besaß die Meter ein Heiligtum in Athen. ${ }^{74}$

Die Historizität dieser Erzählung wird aufgrund ihrer späten Überlieferung und fehlender zeitgenössischer Quellen zu Recht angezweifelt. Einige Forscher_innen vermuten hinter dieser Legende eine Ablehnung der Athener_innen gegen den fremden Kybelekult im ausgehenden 5. und beginnenden 4. Jahrhundert v. Chr. Diese These beruht vor allem auf der negativen Wahrnehmung der Phrygier_innen bei den Griech_innen. Die Phrygier_innen und ihre Kulte wurden als „barbarisch" angesehen und in der Literatur schon bald mit den Nachfahr_innen der Erzfeinde der Griech_innen, den Trojanern, gleichgesetzt. Die Legende wirkt darüber hinaus wie eine typisch mythologische Ätiologie, die erklärt, wie eine fremde Gottheit in das griechische Pantheon aufgenommen wurde und ein Heiligtum in Athen besaß.75

Archäologisch lässt sich das Metroon auf der Agora des 5. Jahrhunderts v. Chr. in Athen nachweisen, das bei Ausgrabungen unter der Leitung des Amerikaners Homer A. Thompson vor dem Zweiten Weltkrieg vollständig freigelegt wurde. ${ }^{76}$ Das Heiligtum der Meter wurde auf dem alten Buleuterion, einem Versammlungsraum des Stadtrates, Ende des 5. Jahrhunderts v. Chr. errichtet. ${ }^{77} 480$ v. Chr. wurde es durch die persische Invasion zerstört, 460 v. Chr. wieder restauriert und auch als Staatsarchiv genutzt.78 267 v. Chr. wurde es erneut zerstört und wieder aufgebaut. ${ }^{79}$ Die zentrale und politisch wichtige Lage des Metroons soll Forschern_innen zufolge auf die Rolle von Kybele als Beschützerin der Demokratie und Rechtsprechung hinweisen sowie als „Hüterin des öffentlichen Gedächtnisses", da in ihrem Heiligtum das Staatsarchiv untergebracht war. ${ }^{80}$

Anderer Meinung ist hingegen Maria Xagorari-Gleißner, die glaubt, das Metroon wäre nicht für Kybele, sondern für Rhea, eine weitere Meter in der griechischen Götterwelt,

\footnotetext{
72 Maarten J. Vermaseren, Cybele and Attis. The Myth and the Cult, London 1977, S. 32.

73 Xagorari-Gleißner, Meter Theon, S. 69.

74 Munn, The Mother of the Gods, S. 58-60.

75 Roller, In Search of God the Mother, S. 166-168.

76 Vermaseren, Cybele and Attis, S. 32.

77 Borgeaud, Die Mutter der Götter, S. 87.

78 Roller, In Search of God the Mother, S. 162

79 Vermaseren, Cybele and Attis, S. 32.

80 Borgeaud, Die Mutter der Götter, S. 87.
} 
gebaut worden. Sie findet es unwahrscheinlich, dass gerade eine fremde Göttin ein Heiligtum und das Staatsarchiv auf einem so zentralen Platz der Agora von Athen erhalten hätte. ${ }^{81}$

Auch wenn noch Zweifel hinsichtlich der Zugehörigkeit des Metroons bestehen, ist das Heiligtum und was sich darin befand für die Ikonographie und die griechische Vorstellung von einer Muttergöttin mit phrygischen Wurzeln von enormer Bedeutung.

\subsection{Ikonographie der Kybele}

Als das bedeutendste Vorbild der griechischen ikonographischen Darstellung von Kybele gilt allgemein das Kultbild der Göttin, welches in ihrem Metroon stand. Im späten 5. Jahrhundert v. Chr. schuf der Bildhauer Agorakritos von Paros diese Statue, von deren Existenz wir nur noch durch schriftliche Aufzeichnungen und zahlreiche kleinere Nachbildungen wissen. ${ }^{82}$ Sie wurde in der klassischen Zeit zum Prototyp der Kybeledarstellungen und blieb bis weit in römische Zeit der Standardtypus der Kybeleikonographie. Sie galt zudem für viele Votive und Statuen als Vorbild mit nur geringen Abweichungen ihrer bekanntesten Motive: Löwe, Tympanon, Opferschale und Mauerkrone.

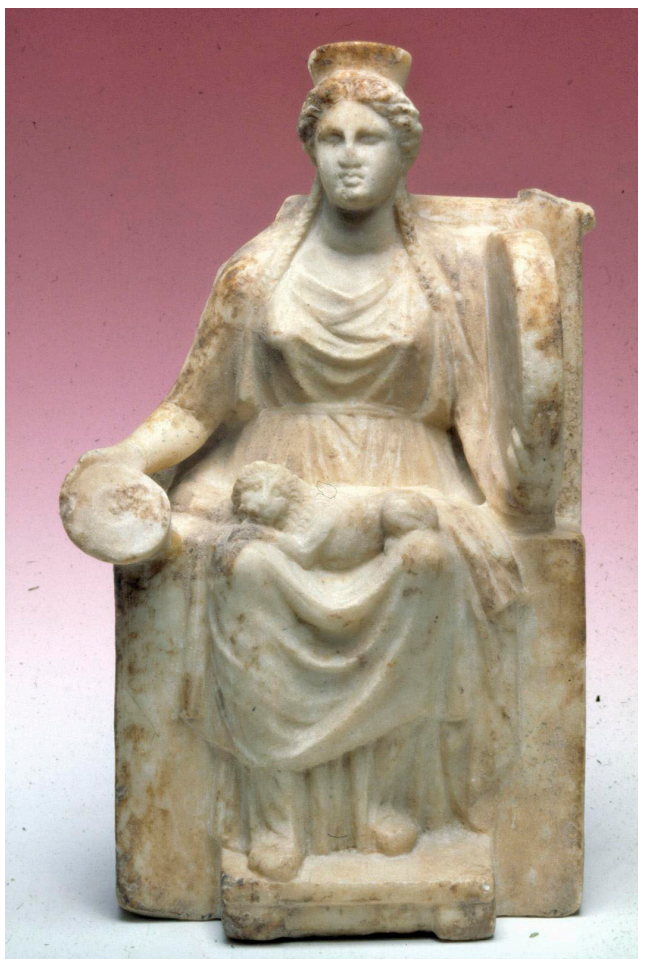

Der Löwe ist wohl das prägendste Symbol für Kybele, in griechischer sowie auch in späterer römischer Zeit. Anhand dieses Begleittieres ist Meter fast immer klar als Kybele zu identifizieren. Doch woher kommt der Löwe, der auch manchmal im Paar mit der Göttin auftritt? Bei den Naiskoi der frühesten Kybeledarstellungen liegt er meist auf dem Schoß der Göttin, bei den späteren Statuen steht der Löwe an ihrer Seite. ${ }^{83}$ Der Löwe kommt in der phrygischen Matarikonographie bis auf das Steinmonument in Arslankaya nicht vor. Matar war zwar die Herrin der Wildnis und wurde daher auch öfter mit wilden Tieren in Verbindung gesetzt, allerdings war der Löwe nur eines von vielen Raubtieren, welche die Muttergöttin begleiteten. Als ursprünglich kennzeichnendes phrygisches Motiv der Matar scheidet der Löwe somit aus. Lynn Roller

Abbildung 1: Miniaturnachbildung der Agorakritos Statue

(Courtesy of the Allard Pierson Museum, Amsterdam).

81 Xagorari-Gleißner, Meter Theon, S. 24.

82 Takacs, Kybele, Sp. 952; Roller, In Search of God the Mother, S. 143.

83 Xagorari-Gleißner, Meter Theon, S. 36. 
vertritt die Meinung, dass der Löwe eine lydische Beifügung war. Der Löwe war ein mächtiges Symbol des lydischen Königshauses. Durch die Aufnahme von Löwen in die Ikonographie von Kybebe/Kybele sei eine Verbindung zwischen Herrscher und Göttin entstanden. Damit wurde der König unter den göttlichen Schutz der Muttergottheit gestellt. Löwen waren darüber hinaus ein beliebtes Motiv in lydischen Kultobjekten und dieses lydische Motiv sei, so meint Roller, von den Griech_innen aufgenommen worden. ${ }^{84}$ Warum also ist der Löwe für Kybele ein so charakteristisches Attribut geworden? Brigitte Bøgh verweist darauf, dass die phrygische Matar in ihrem Heimatland eine Art Monopolstellung unter den Gottheiten hatte und deshalb nicht genauer definiert werden musste. ${ }^{85}$ In Griechenland aber trifft die Göttermutter nun auf eine Reihe schon vorhandener Muttergottheiten. Um sie von den anderen zu unterscheiden, hätte Kybele eine eigene ikonographische Identität gebraucht. Der Löwe wurde somit zum eigentlichen Identifikationsmerkmal der Kybele.

Allerdings war Kybele nicht die einzige Göttin, die mit einem Löwen dargestellt wurde und es gibt Hinweise, dass andere Abbildungen von weiblichen Gottheiten mit Löwen das Kybelebild und ihre Motive beeinflussten. Eine bronzezeitliche, minoische, noch nicht identifizierte Göttin findet sich beispielsweise auf Siegeln. Darauf ist eine stehende weibliche Figur auf einer Bergspitze, flankiert von zwei Löwen, abgebildet. ${ }^{86}$ Weiters ist auch Potnia Theron, die Herrin der Wildnis und wilden Tiere, öfter mit Löwen in ihrer Begleitung dargestellt. ${ }^{87}$ Desgleichen sind auch Bildnisse von Artemis und Hera in Gesellschaft von Löwen bekannt. ${ }^{88}$ Es kann also nicht ausgeschlossen werden, dass das schon vorhandene griechische Motiv des Löwen mit dem lydischen Löwen von Kybebe/Kybele verbunden wurde.

Das Tympanon war ein weiteres Charakteristikum, welches die Griech_innen der Göttin in der Mitte des 5. Jahrhunderts hinzufügten. ${ }^{89}$ Das Trommelinstrument hielt die Göttin meist in der linken Hand und in der rechten eine Opferschale. Das Tympanon stammte aus dem Osten und wurde unter anderem für Kultrituale im assyrischen und neohethitischen Raum benutzt, es gibt allerdings noch keine Hinweise dafür, dass es auch im phrygischen Raum bekannt war. ${ }^{90}$ Es stellt sich daher, genau wie beim Löwenmotiv, die Frage, weshalb es zu einem von Kybeles wichtigsten Erkennungsmerkmalen wurde. Eine naheliegende Erklärung ist die Bedeutung, die das Instrument und die Opferschale für den Kult der Kybele hatten. Daraus ließe sich schlussfolgern, dass diese Objekte nicht für die Göttin persönlich kennzeichnend waren, sondern für ihre Kultanhänger_innen und deren Praktiken eine wichtige Funktion hatten.91 Die Mauerkrone von Kybele erlangte besonders in römischer Zeit, in ihrer Funktion als Schutzgöttin der Stadt, eine größere Bedeutung. Die Mauerkrone war zwar ein bekanntes Motiv aus

\footnotetext{
84 Roller, In Search of God the Mother, S. 131.

85 Bøgh, The Phrygian Background, S. 325.

86 Roller, In Search of God the Mother, S. 134.

87 Ebd., S. 135.

88 Xagorari-Gleißner, Meter Theon, S. 36.

89 Ebd., S. 38

90 Roller, In Search of God the Mother, S. 136-137.

91 Ebd., S. 148.
} 
dem anatolischen und phrygischen Raum, Matar selbst aber wurde nie mit ihr dargestellt.92

Diese Attribute, Löwe, Tympanon, Schale und Mauerkrone, wurden in den darauffolgenden Jahrhunderten zu den wesentlichen Merkmalen von Kybele. Durch sie konnten die Griech_innen sie als eine fremde, phrygische Göttin erkennen, gleichzeitig aber stammten diese Objekte ursprünglich nicht aus Phrygien. Die Kennzeichen, die sie als "wilde" phrygische Göttin auszeichneten, wurden erst von den Griech_innen selbst hinzugefügt. Die Griech_innen übernahmen also keine genuinen Attribute von Matar, sondern entwickelten eine eigene Vorstellung von einer phrygischen Göttin und deren Attributen, welche sie dann auf ihre Meter anwandten. ${ }^{93}$

Über die Ikonographie der Kybele kann zumindest teilweise auf ihre Kultrituale geschlossen werden. Wie wurde sie aber von den Griech_innen im öffentlichen und privaten Rahmen verehrt? Die Kultpraktiken und Mysterien zu Kybele geben uns nach wie vor viele Rätsel auf. Daher ist es lohnenswert, als Nächstes das vorhandene Material und divergierende Interpretationsansätze der Forscher_innen zu diesem Thema aufzugreifen und zu untersuchen.

\section{Kybeles Aufnahme in Griechenland}

\subsection{Kult und Mysterien}

Über den frühen Kult zu Kybele in Griechenland gibt es nur wenige aussagekräftige Quellen. Vieles, das uns erhalten ist, stammt aus den Federn von späteren römischen und griechischen Autoren, die meist mit einer negativen Haltung vor allem über die Mysterien berichteten. ${ }^{94}$

Es gibt überschaubare überlieferte Quellen, die uns über die Kultpraktiken oder das Kultpersonal informieren und nicht aus der Zeit des Hellenismus oder der römischen Kaiserzeit stammen. Das Metroon und die Kultstatue für die Göttermutter belegen zumindest eine offizielle Einführung von Kybele in das griechische Pantheon und somit auch in den öffentlichen Götterkult der Polis. Nicht nur in Athen hatte die Göttermutter ein Heiligtum, sondern bald auch in anderen Städten. Der Staatsmann Themistokles gründete einen Kult für die Mutter der Götter in Magnesia als Dank dafür, dass sie ihm in einem Traum erschienen war und ihn vor einem geplanten Attentat gewarnt hatte. Auch in Olympia wurde im Zeusheiligtum ein Metroon errichtet. ${ }^{95}$ Tempel und Kultstätten, die für die breite Öffentlichkeit zugänglich waren, sprechen für eine offizielle anerkannte Kultausübung, die auch von den Stadtherrschern mitgetragen wurde. So meint Philipp Borgeaud, dass die griechische Meter einen wichtigen Kultritus für Demokratie und Rechtsprechung inne hatte. ${ }^{96}$

92 Bøgh, The Phrygian Background, S. 309.

93 Roller, In Search of God the Mother, S. 149.

94 Ebd

95 Roller geht allerdings von einem Heiligtum für Rhea/Meter aus; Roller, In Search of God the Mother, S. 161.

96 Borgeaud, Die Mutter der Götter, S. 87. 
Im privaten Rahmen zeugen die vielen Votivgaben und Statuetten von einer hohen Popularität der Göttermutter bei der Bevölkerung. Diese archäologischen Funde geben auch manchmal Auskunft über die Glaubensanhänger_innen und über die Gründe, aus welchen sie sich an Kybele wandten. Die Masse der Votive ist jedoch anonym und es kann nur auf einige mit Inschriften versehenen Schenkungen und Opfergaben zurückgegriffen werden. Die unterschiedliche Qualität bei den Votivgaben aus dem 5. und 4. Jahrhundert legen die Vermutung nahe, dass Kybeleanhänger_innen aus verschiedenen sozialen Schichten stammten. ${ }^{97}$ Die Göttermutter sprach im Übrigen beide Geschlechter an, Frauen etwas mehr als Männer, wie sich aus den Funden schließen lässt. ${ }^{98}$ Das Kultpersonal beinhaltete sowohl weibliche, als auch männliche Priester_ innen für den Kybelekult. Für Eunuchen, den kastrierten Priestern der Göttermutter, scheint es hingegen für Griechenland in vorrömischer Zeit keine Evidenz zu geben. ${ }^{99}$ Diese Priester waren erst im römischen Kult der Magna Mater sicher belegt. ${ }^{100}$ Ansonsten geht die Quellenlage sehr spärlich mit Informationen über die Kultausübungen zu Kybele um. Das gilt vor allem in Bezug auf die Mysterien von Kybele.

Wie alle Mysterienkulte der Antike waren auch die Mysterien der Göttermutter streng geheim und nur für Eingeweihte zugänglich. Für Außenstehende war es deshalb verständlicherweise schwierig, klare Aussagen über diesen Kult zu treffen. Forscher_innen des 19. und 20. Jahrhunderts, wie etwa Paul Foucart, Franz Bömer und Hendrik Simon Versnel, zogen meist römische und christliche Autoren als Quellen heran, die jedoch zu einer eher negativen Tendenz in der Beschreibung der Mysterienkulte neigten. Darüber hinaus sprachen diese antiken Autoren mehrheitlich von den römischen Kulten und die Forscher_innen übertrugen diese tendenziösen Berichte auf die griechischen Kulte der klassischen Zeit. ${ }^{101}$ Aus griechischen Schriften und Abbildungen lässt sich nur so viel herauslesen: Die kultischen Riten fanden nachts statt, begleitet von Fackeln, Musik und Tanz. ${ }^{102}$

Ein Krater aus Spina aus dem frühen 5. Jahrhundert v. Chr. lässt die Forscher_innen rätseln, ob die Darstellungen eine getreue Wiedergabe eines solchen Mysterienrituals sind. Auf der rotfigurigen attischen Vase sind auf einer Seite zwei sitzende Figuren in einem abgetrennten Bereich zwischen zwei dorischen Säulen dargestellt. Die weibliche Figur kann durch den Löwen und die Opferschale als Kybele identifiziert werden. Bei der männlichen Figur scheint es sich ebenfalls um einen Gott zu handeln, wobei nicht ganz klar ist, um welchen. Kybele wird gewöhnlich mit mehreren männlichen Göttern dargestellt, sei es Attis, ihrem Geliebten, Pan oder Dionysos. ${ }^{103}$ Weitere Figuren unterschiedlichster Größe, die tanzen und musizieren, sind auf den beiden Seiten der Vase zu sehen. Allein diese Szenen lassen verschiedene Interpretationen für den Mysterienkult der Göttin zu. Wurden kultische Riten oder ekstatische Tänze, welche die

97 Roller, In Search of God the Mother, S. 182.

98 Xagorari-Gleißner, Meter Theon, S. 69.

99 Ebd., S. 68.

100 Bøgh, The Phrygian Background, S. 323.

101 Roller, In Search of God the Mother, S. 149

102 Ebd.

103 Ebd., S. 176. 
Anhänger_innen in einen tranceähnlichen Zustand versetzten, abgebildet? Die Szene auf der Vase bildete Anlass für eine Vielzahl von Spekulationen unter den Forscher_innen. Roller geht davon aus, dass diese Abbildung durchaus eine reale Vorstellung der Zeitgenoss_innen von den Mysterien war. Sie schließt dabei aber nicht aus, dass es sich auch um eine sarkastische Interpretation von Außenstehenden handeln könnte, für welche das Treiben der Eingeweihten unverständlich sein musste. ${ }^{104}$ Xagorari-Gleißner hingegen vertritt die Meinung, auf dem Krater sei ein orphischer Kult der Meter und des Dionysos abgebildet. Zudem meint sie, Meter trage das Zepter der Demeter sowie das Diadem von Hera und wäre somit eine Verschmelzung dieser beiden Göttinnen. Das wiederum würde zu den Aussagen des „Derveni-Papyrus“ passen, in denen Meter mit vielen anderen griechischen Göttinnen gleichgesetzt wurde. ${ }^{105}$

Meter oder Kybele wurde seit ihrer Ankunft in der griechischen Welt mit anderen Göttinnen in unterschiedlicher Art und Weise gleichgesetzt oder identifiziert. Inwieweit das für eine Akzeptanz von Kybele in der griechischen Götterwelt hilfreich wurde, wird im abschließenden Teil dieser Arbeit besprochen werden.

\subsection{Kybele im griechischen Pantheon}

Nach ihrer Ankunft in Griechenland wurde Kybele recht bald mit einheimischen weiblichen Göttinnen gleichgesetzt. ${ }^{106}$ Es war dabei äußerst hilfreich, dass sie mit einigen Göttinnen viele Aspekte gemeinsam hatte und für die Griech_innen stellte dieser Synkretismus von zwei ansonsten unterschiedlichen Göttinnen nichts Ungewöhnliches dar. Kybeles Status als fremde Gottheit konnte unter anderem durch Identifikationsmöglichkeiten mit griechischen Göttinnen abgemildert werden. Drei Muttergöttinnen spielten dabei eine besondere Rolle.

Gaia war die Personifikation der Erde, die Mutter Erde und allen Lebens. Im griechischen Verständnis blieb sie allerdings eine abstrakte Vorstellung und auch in bildlichen Darstellungen war sie selten aufzufinden. ${ }^{107}$ Viel verständlicher ist daher die Identifikation mit Rhea. Rhea war die Mutter der sechs olympischen Gottheiten und damit selbst eine Mutter der Gottheiten. Mit der Gleichsetzung von Rhea wurde nun auch Kybele zur Mutter der griechischen Gottheiten erhoben. Es zeigt sich auch eine sprachliche Ähnlichkeit zwischen dem Titel der Kybele, Meter oreia, und dem Namen Rhea. Eine weitere Parallele sind die Kultorte der Göttinnen. Es gab einen Rhea- und Zeuskult auf dem Berg Ida in Kreta, auf dem der mythologischen Erzählung zufolge Rhea ihren Sohn Zeus vor seinem Vater Kronos versteckte. ${ }^{108}$ Auch die phrygische Matar hatte einen Kultort auf einem Berg namens Ida in Nordwestanatolien. Bei den Römer_innen wurde sie als die "Mater Deum Magna Idaea“109, die Große Mutter vom Berg Ida, bekannt.

\footnotetext{
104 Roller, In Search of God the Mother, S. 151-155.

105 Xagorari-Gleißner, Meter Theon, S. 63.

106 Takacs, Kybele, Sp. 952.

107 Roller, In Search of God the Mother, S. 170.

108 Lutz Käppel, Rhea, Rheia, in: Neue Pauly 2006, [http://dx.doi.org/10.1163/1574-9347_dnp_e1021870], eingesehen 4.8.2016.

109 Takacs, Kybele, Sp. 953.
} 
Zudem hatte Rhea in Griechenland keinen ausgeprägten Kult und ihre Person war im Vergleich zu den bekannteren griechischen Gottheiten eher blass und unscheinbar. Durch einen Synkretismus mit der nicht-griechischen Kybele wurde somit kein schon vorhandener Kult verdrängt und Kybele ließ sich leichter integrieren. Zuletzt sind noch die auffallenden Ähnlichkeiten bei den Kultriten der beiden Göttinnen zu nennen. Rhea wurde auch mit ekstatischen Tänzen, Musik und lauten Geräuschen bei ihren Ritualen in Verbindung gebracht. ${ }^{110}$ Die beiden Göttinnen hatten also durchaus Gemeinsamkeiten.

Eine weitere Verbindung bestand zwischen der griechischen Demeter und Kybele. Die beiden Göttinnen wurden seit dem 6. Jahrhundert v. Chr. miteinander in Beziehung gebracht. In diesem Fall ist es interessant zu sehen, dass beide Göttinnen aus ikonographischer Sicht aufeinander eingewirkt hatten. Es gibt Demetervotive, die sie auf einem Thron sitzend mit einem Löwen auf dem Schoß darstellen. Die griechische Göttin hat somit deutlich typische Kybelemotive übernommen. Diese beiderseitige Übernahme von Symbolen verstärkte sich in den folgenden Jahrhunderten noch. ${ }^{111}$ Die Szene auf dem Krater aus Spina, in der Kybele der Interpretation Xagorari-Gleißners zufolge, das Zepter der Demeter in der Hand hält, lässt sich somit als eine Übernahme von Demetermotiven durch Kybele deuten.

Diese drei Göttinnen, Gaia, Rhea und Demeter, standen also in mehr oder weniger ausgeprägter Verbindung zu Kybele. Einige Forscher_innen sehen in diesem Synkretismus der Göttinnen einen aktiven Versuch der Griech_innen, diese „wilde“ phrygische Göttin zu domestizieren, um ihre Person und ihren Kult für die Öffentlichkeit akzeptabler zu machen. ${ }^{12}$ Dabei wurden ihre vermeintlich phrygischen und "wilden" Motive allesamt von den Griech_innen selbst hinzugefügt. In jedem Fall gelang die Integration von Kybele in das griechische Pantheon wesentlich leichter mit Verbindungen zu einheimischen Gottheiten. Kybele war zwar keine Hauptgöttin der Griech_innen, aber sie stand auch nicht völlig am Rande, wie die hohe Anzahl an Votivgaben an ihren Kultorten aufzeigt.

Die Ambivalenz in Kybeles Wesen, einmal die besondere Aufmerksamkeit auf ihre nicht-griechischen Wurzeln und die Hervorhebung ihrer Stellung als Göttermutter, schienen der Popularität der Göttin nicht geschadet zu haben. In hellenistischer und römischer Zeit blieb sie für lange Zeit bekannt und genoss weitverbreitete Popularität.

\section{Resümee}

In dieser Arbeit wurden die Herkunft, Reise und die wichtigsten Veränderungen auf dem Weg der Göttin Kybele nach Griechenland herausgearbeitet. Aus dem archäologischen Fundmaterial in Phrygien lässt sich eine Muttergöttin, die eine Vorgängerin der griechischen Kybele war, erkennen. Die Forschung hat indessen, mangels eindeutiger

\footnotetext{
110 Roller, In Search of God the Mother, S. 170-173.

111 Ebd., S. 174

112 Ebd., S. 175.
} 
Quellen, die unterschiedlichsten Vorstellungen zu Matars Herkunft und Abstammung hervorgebracht. Dabei wurde lediglich festgestellt, dass es zwar ausreichend Thesen und Theorien gibt, aber keine darunter den vollen Konsens der Forscher_innen erhält.

Aus dem vorhandenen Quellenmaterial, sei es schriftlicher oder materieller Natur, wurde der Weg der Göttin von den kleinasiatischen Küstenstädten bis nach Athen nachgezeichnet, wo die Göttermutter im 5. Jahrhundert v. Chr. schließlich ankam. Schriftliche Quellen von den Griech_innen belegen die Kenntnis einer Muttergottheit phrygischer Abstammung. Die wohl bedeutendsten Zeugnisse für einen vorhandenen Kybelekult bilden allerdings die zahlreichen Votivgaben an die Göttin in den kleinasiatischen Küstenstädten. Die Bilder, die sich die Griech_innen von der fremden, in die eigene Götterwelt aufgenommenen Kybele machten, bezeugen vielmehr deren Eigenansichten und Akzeptanz gegenüber der Gottheit. Die Attribute in der ikonographischen Darstellung geben uns wertvolle Hinweise auf das Verhältnis der Bevölkerung zur Göttin. Ihre phrygischen Wurzeln wurden trotz Assimilationsversuchen mit einheimischen Muttergöttinnen nicht vergessen und auch nicht vollständig verdrängt. Das Gegenteil war der Fall: Vermeintlich phrygische Attribute, wie Tympanon und der lydische Löwe, wurden zu den herausragenden Merkmalen der Göttin, die es ermöglichten, sie eindeutig als griechische Kybele mit phrygischen Wurzeln zu identifizieren. Der Synkretismus mit einheimischen Göttinnen war ein Versuch, Kybele für die Griech_innen akzeptabler zu machen. Die Muttergöttin sprach eine breite Masse der Bevölkerung an, was vermutlich auch ein Grund für ihren langlebigen und weitverbreiteten Kult war. Die wandelbare Vorstellung der Kybele in Griechenland macht deutlich, wie die Griech_innen eine fremde Göttin aufnahmen und für die eigenen religiösen Bedürfnisse anpassen konnten, ohne ihre nicht-griechische Herkunft zu verdrängen.

\section{Literatur}

Bachofen, Johann Jakob, Das Mutterrecht. Eine Untersuchung über die Gynaikokratie der alten Welt nach ihrer religiösen und rechtlichen Natur, Stuttgart 1861.

Betegh, Gabor, The Derveni Papyrus. Cosmology, Theology and Interpretation, Cambridge 2005.

Bittel, Kurt, Kubaba, in: Reallexikon der Assyriologie und Vorderasiatischen Archäologie, Bd. 6, Berlin-New York 1983, Sp. 257-265.

Bøgh, Brigitte, The Phrygian Background of Kybele, in: Numen 54 (2007), Heft 3, S. $304-$ 339, [http://www.jstor.org/stable/27643268], eingesehen 12.7.2016.

Borgeaud, Philippe, Die Mutter der Götter. Von Anatolien über Griechenland nach Rom, in: Badisches Landesmuseum Karlsruhe (Hrsg.), Imperium der Götter. Isis-Mithras-Christus, Kulte und Religionen im Römischen Reich, Darmstadt 2013, S. 85-91.

Graf, Fritz, The Arrival of Cybele in the Greek east, in: János Harmatta (Hrsg.), Acts du VIle Congrès de la Fédération Internationale des Associations d’Études Classiques I, Budapest 1984, S. 117-120. 
Högemann, Peter, Midas, in: Der Neue Pauly 2006, [http://dx.doi.org/10.1163/15749347_dnp_e803950], eingesehen 4.8.2016.

Käppel Lutz, Rhea, Rheia, in: Der Neue Pauly 2006, [http://dx.doi.org/10.1163/15749347_dnp_e1021870], eingesehen 4.8.2016.

Kippenberg, Hans G. (Hrsg.), Bachofen, Johann Jakob. Mutterrecht und Urreligion, Stuttgart 1984.

Munn, Mark, The Mother of the Gods, Athens, and the Tyranny of Asia. A Study of Sovereignty in Ancient Religion, Berkeley-Los Angeles-London 2006.

Naumann, Friederike, Die Ikonographie der Kybele in der phrygischen und der griechischen Kunst (Istanbuler Mitteilungen Beiheft 28), Tübingen 1983.

Olshausen, Eckart, Phryges, Phrygia, in: Der Neue Pauly, Bd. 6, Stuttgart-Weimar 1999, Sp. 965-967.

Rein, Mary Jane, Phrygian Matar. Emergence of an Iconographic Type, in: Eugene N. Lane (Hrsg.), Cybele, Attis and Related Cults. Essays in Memory of M. J. Vermaseren (Religions in the Graeco-Roman World 131), Leiden-New York-Köln 1996, S. 223-237.

Roller, Lynn E., In Search of God the Mother. The Cult of Anatolian Cybele, Berkley-Los Angeles-London 1999.

Takacs, Sarolta A., Kybele, in: Der Neue Pauly, Bd. 6, Stuttgart-Weimar 1999, Sp. 950-956. Vassileva, Maya, Further Considerations on the Cult of Kybele, in: Anatolian Studies 51 (2001), S. 51-63, [http://www.jstor.org/stable/3643027], eingesehen 11.7.2016.

Vermaseren, Maarten J., Cybele and Attis. The Myth and the Cult, London 1977.

Wesel, Uwe, Der Mythos vom Matriarchat. Über Bachofens Mutterrecht und die Stellung von Frauen in frühen Gesellschaften vor der Entstehung staatlicher Herrschaft, Frankfurt am Main 1981.

Wittke, Anne-Maria, Kleinasien, in: Der Neue Pauly 2006, [http://dx.doi.org/10.1163/ 1574-9347_dnp_e614980], eingesehen 4.8.2016.

Wolf, Erik/Bachofen, Johann Jakob, in: Deutsche Biographie, o. D., [https://www.deutsche-biographie.de/gnd1 18505645.html\#ndbcontent], eingesehen 27.3.2018.

Xagorari-Gleißner, Maria, Meter Theon. Die Göttermutter bei den Griechen (Peleus Studien zur Archäologie und Geschichte Griechenlands und Zyperns 40), Mainz-Ruhpolding 2008.

\section{Quellen}

Diodorus Siculus, Griechische Weltgeschichte (Bibliothek der griechischen Literatur 34), Buch I-X. Teil 1: Buch I-III, übersetzt und herausgegeben von Gerhard Wirth/Otto Veh, Stuttgart 1992. 
Euripides, Bacchae, The Plays of Euripides, Bd. 9, Griechisch-Englisch, übersetzt und eingeleitet von Richard Seaford, herausgegeben von Christopher Collard, Warminster 1996.

Herodot, Historien, Griechisch-Deutsch, herausgegeben von Josef von Feix (Sammlung Tusculum), Düsseldorf 2006.

Homer, Homerische Hymnen, Griechisch-Deutsch, herausgegeben von Anton Weiher (Sammlung Tusculum), München-Zürich 1986.

Pindar, Siegesgesänge und Fragmente, Griechisch-Deutsch, herausgegeben von Oskar Werner, München 1967.

\section{Abbildungsverzeichnis}

Abbildung 1: Miniaturnachbildung der Agorakritos Statue (Courtesy of the Allard Pierson Museum, Amsterdam).

Sabine Viktoria Kofler ist Studentin der Geschichtswissenschaften (MA) an der Universität Innsbruck. Sabine.Kofler@student.uibk.ac.at

\section{Zitation dieses Beitrages}

Sabine Viktoria Kofler, Kybele in Griechenland - Ankunft und Aufnahme der Göttermutter in der griechischen Welt, in: historia.scribere 10 (2018), S. 379-399, [http://historia. scribere.at], eingesehen 19.6.2018 (=aktuelles Datum).

(C) Creative Commons Licences 3.0 Österreich unter Wahrung der Urheberrechte der Autorlnnen. 
